\title{
PO3-2-26
}

Poster session

\section{The Use of Non-steroidal Anti-inflammatory Drugs in an Australian Elite Athlete Population}

\author{
Alison Shield ${ }^{1}$, Thomas Eason $^{1}$, Julie Cooke $^{2}$ \\ ${ }^{1}$ Pharmacy, University of Canberra, Australia, ${ }^{2}$ Sport and Exercise Science, University of Canberra, Australia
}

Background

Non-steroidal anti-inflammatory medications (NSAIDs) are reported to be highly used by elite athletes, and their knowledge regarding the proper indications and side effects is often poor. Identifying areas where these athletes lack knowledge could lead to interventions to improve their health outcomes and minimize the risks associated with these medications. The major purpose of this study was to determine how a representative sample of Australian elite athletes is using NSAIDs and their knowledge and perceptions of these medications.

Methods

An online survey with participant information was distributed to physically active adults for completion.

Results

A total of 379 participants completed the survey; 80 elite or professional athletes completed the survey, had utilized NSAID medication within the prior 6 months and were included in further analysis. The majority of participants (85\%) used NSAIDs for the evidence-based indication of treating an acute injury, and 78\% followed the instructions on the packaging regarding dosage. However, the athletes were unclear as to whether NSAIDs should be used prophylactically or to treat delayed onset muscle soreness (DOMS). They also lacked knowledge regarding side effects, with $30 \%$ suggesting liver damage as a side effect and $26 \%$ suggesting constipation, both of which are incorrect responses. Eleven percent of athletes reported having experienced an adverse reaction to NSAID use, although up to $30 \%$ admitted to knowing no side effects of NSAIDs. Only $52 \%$ perceive additional risk with using multiple NSAIDs concurrently.

Conclusions

There was high use of NSAIDs among the participants, with most utilizing them for acute injury healing. Their perceptions and knowledge of side effects showed a general lack of knowledge regarding how these medications should be used. Improving their knowledge in these areas would reduce the risk of inappropriate use of these medications and reduce their risk of side effects. 